\title{
Percepción y conocimiento de la biodiversidad por estudiantes urbanos y rurales de las tierras áridas del centro-oeste de Argentina
}

\author{
Claudia M. Campos ${ }^{1, \otimes}$; Juliana Nates ${ }^{2} \&$ Petra Lindemann-Matthies $^{3}$ \\ 1. Grupo de Investigaciones de la Biodiversidad (IADIZA-CONICET, Mendoza) e INTERBIODES (UNSJ-CONICET, \\ San Juan), Argentina. 2. Institute of Evolutionary Biology and Environmental Studies, University of Zürich, Switzerland. \\ 3. Institute of Biology, University of Education, Karlsruhe, Germany.
}

\begin{abstract}
RESUMEN. Los ecosistemas áridos y semiáridos de las provincias de Mendoza y San Juan en la actualidad sufren una degradación fuerte, con pérdida de biodiversidad. En este escenario, la difusión y la educación acerca de la biodiversidad nativa y su valor resultan indispensables a los fines de conservación. El presente trabajo revisa tres estudios llevados a cabo en las provincias de Mendoza y San Juan. Estos estudios fueron los primeros que, por un lado, intentaron reflejar las percepciones, conocimientos y apreciaciones de los estudiantes urbanos y rurales acerca de la biodiversidad y, por el otro, presentaron los resultados de la evaluación de una intervención educativa orientada a mejorar conocimientos y actitudes hacia la biodiversidad. Los trabajos muestran que si bien la procedencia, el género y la edad de los estudiantes afectan la percepción y el conocimiento acerca de la biodiversidad, en general, los niños aprecian y conocen más a las especies exóticas que a las nativas y, como en el resto del mundo, están familiarizados con las mascotas, las plantas ornamentales y los mamíferos carismáticos. Los estudiantes aprenden sobre biodiversidad utilizando diferentes fuentes; el contacto inicial de los más jóvenes con las especies ocurre a través de los medios masivos de comunicación y los libros. Los niños están poco familiarizados con las especies nativas locales con problemas de conservación y conocen poco acerca de sus adaptaciones evolutivas a las zonas áridas. Luego de una corta intervención educativa se observan cambios en percepciones y conocimientos en el corto plazo, pero a largo plazo se pierden. Es importante aumentar el esfuerzo en las escuelas, la familia, las organizaciones no gubernamentales y otros para mejorar el conocimiento y la apreciación de las biodiversidad nativa a través de actividades que se mantengan en el tiempo e impliquen el contacto directo con la naturaleza.
\end{abstract}

[Palabras clave: apreciación, actitudes, especies nativas, especies exóticas]

\begin{abstract}
Aвstract. Perception of and knowledge about biodiversity by urban and rural students of central-west drylands of Argentina: Arid and semiarid ecosystems of Mendoza and San Juan are currently undergoing severe degradation with the subsequent loss of biodiversity. In this scenario, diffusion and education about native biodiversity and its value are indispensable to the purposes of conservation. The present work reviews three previous studies conducted in the provinces of Mendoza and San Juan. This review attempts to reflect the perceptions, knowledge and appreciation of urban and rural students about biodiversity, within the framework of studies conducted worldwide, and discusses the effect of educational interventions aimed at improving knowledge of and attitudes toward biodiversity. Studies find that, although the place of residence, sex and age of students affect their perception and knowledge of biodiversity, overall, children appreciate and know exotic species better than native ones and, just as in the rest of the world, they are familiar with pets, ornamental plants and charismatic mammals. Students learn about biodiversity from different sources; the initial contact of the youngest children with species is through the mass media and books. Children are scarcely familiar with local native species with conservation issues or with their evolutionary adaptations to arid regions. After a short educational intervention, changes in perceptions and knowledge are observed in the short term, but these are lost in the long term. We consider it important to increase efforts in schools, family, non-governmental organizations, etc., to improve knowledge and appreciation of native biodiversity through activities that are maintained over time and involve direct contact with nature.
\end{abstract}

[Keywords: appreciation, attitudes, native species, exotic species]

\section{INTRODUCCIÓN}

Las tierras áridas de las provincias de San Juan y Mendoza incluyen zonas hiperáridas, áridas, semiáridas y subhúmedas secas. En la antigüedad, estos ecosistemas, caracterizados por su rigurosidad climática, albergaron poblaciones humanas que basaron su subsistencia en la recolección de frutos y

$\triangle$ ccampos@mendoza-conicet.gob.ar. la caza de animales silvestres (Vilela et al. 2009). A fines del siglo XIX tuvieron lugar transformaciones importantes del paisaje debidas al desarrollo de sistemas de riego que permitieron la expansión de la agricultura en los llamados "oasis" (Abraham et al. 2009). En la actualidad, estos oasis ocupan porciones pequeñas del territorio, pero concentran la 
mayor parte de la población y de los recursos económicos, mientras las zonas no irrigadas mantienen índices de ruralidad altos y basan su subsistencia sobre actividades relacionadas a la ganadería (Abraham et al. 2009).

Los hábitats sufrieron una degradación fuerte -con la consiguiente pérdida de biodiversidad- después de siglos de uso intensivo de sus recursos naturales (Tabeni \& Ojeda 2005; Vilela et al. 2009). Varias especies de animales nativos están incluidos en categorías de conservación de Listas Rojas y apéndices de CITES (Convention on International Trade in Endangered Species of Wild Fauna and Flora), como el guanaco (Lama guanicoe), el cóndor (Vultur gryphus), la mara (Dolichotis patagonum), la vizcacha de la sierra (Lagidium viscacia), las ratas cola de pincel (Tympanoctomys barrerae y Octomys mimax), el pichiciego (Chlamyphorus truncatus), los ñandúes (Rhea americana y Pterocnemia pennata) y la tortuga terrestre (Chelonoidis chilensis) y boa (Boa constrictor) (García Fernández et al. 1997; Ojeda \& Díaz 1997; Díaz \& Ojeda 2000; Barquez et al. 2006; Giraudo et al. 2012; Prado et al. 2012; CITES), y son protegidos por leyes de regulación (Vilela et al. 2009). Las plantas nativas, utilizadas tradicionalmente como fuente de alimento, leña y medicinas (Ladio \& Lozada 2009; Vilela et al. 2009), se encuentran amenazadas por la tala, la expansión del cultivo de especies exóticas y el sobrepastoreo por el ganado doméstico.

Para alcanzar objetivos de conservación ante un escenario de pérdida continua de hábitats y de biodiversidad no sólo son necesarias medidas como el establecimiento de áreas protegidas, regulaciones legales que permitan el uso de los recursos naturales y el control de las especies introducidas, también son fundamentales la difusión y la educación acerca de la biodiversidad nativa y su valor. Además, también es importante difundir las consecuencias potenciales de las perturbaciones provocadas por las actividades humanas (Pimbert \& Pretty 1995; Colton \& Alpert 1998; Trombulak et al. 2004).

Las actitudes de las personas hacia las especies definen en gran parte el comportamiento que tendrán hacia la biodiversidad. Estas actitudes son patrones o marcos de referencia que contribuyen de forma decisiva a la organización del universo cognitivo, permiten categorizar la información que llega en forma de nuevas experiencias y ayudan a simplificar y comprender el complejo mundo en que vivimos. Las actitudes facilitan las tareas de decisión y sirven de guía de acción en cada caso, por lo que ofrecen una pauta de conducta estable (Schacter et al. 2011). Las percepciones, las valoraciones y los conocimientos son algunos de los factores que afectan a las actitudes. Por este motivo, lo que las personas sienten, creen y conocen acerca del ambiente es lo que determina sus actitudes hacia él (Pooley \& O'Connor 2000).

El presente trabajo conforma una revisión de tres estudios previos llevados a cabo en las provincias de Mendoza y San Juan. Estos estudios fueron los primeros que, por un lado, intentaron reflejar las percepciones, conocimientos y apreciaciones de los estudiantes urbanos y rurales acerca de la biodiversidad y, por el otro, mostraron los resultados dela evaluación de una intervención educativa orientada a mejorar conocimientos y actitudes hacia la biodiversidad. La presente revisión analiza los resultados de los trabajos previos antes mencionados en el marco de estudios llevados a cabo en el mundo.

\section{PercepCión de LA Biodiversidad}

La percepción ha sido definida como la identificación, interpretación y organización de las sensaciones para producir una experiencia significativa acerca del mundo (Schacter et al. 2011). Este proceso involucra tanto información sensitiva como preferencias innatas y experiencias previas. Determinadas características físicas y comportamentales de los organismos afectan las actitudes que las personas tienen hacia ellos (Kellert 1993; Wandersee \& Schussler 2001). El atractivo estético, la utilidad o la rareza pueden ser atributos que influencien la opinión de las personas. Aquellas especies que son percibidas como más importantes o son más apreciadas que otras, con seguridad serán las que reciban más apoyo en la formulación y la ejecución de planes de conservación (Czech et al. 1998; Kellert 1996; Montgomery 2002; Tisdell et al. 2005). Incluso, estas percepciones y apreciaciones positivas acerca de ciertas especies pueden resultar un gran obstáculo cuando van dirigidas hacia especies exóticas invasoras que se pretende controlar o erradicar (Veitch \& Clout 2001). Por esto, el estudio de las percepciones acerca de la biodiversidad por diferentes actores resulta crucial a la hora de tomar decisiones de conservación y manejo de especies. 
Tanto los niños como los adultos reconocen y aprecian más a los animales que a las plantas (Flannery 1991; "plant blindness" según Wandersee \& Schussler 2001). Los niños están más informados acerca de los animales (Ryman 1974; Hershey 1996; LindemannMatthies 2002), prefieren aprender sobre animales en la escuela (Baird et al. 1984) y se preocupan más por la conservación de la fauna (Greaves et al. 1993; Ashworth et al. 1995). Probablemente los niños se interesen más en los animales por su movilidad (Wandersee 1986; Kinchin 1999), porque son capaces de establecer contacto visual y comunicarse a través de sonidos (Wandersee 1986), o porque tienen comportamientos que resultan más atractivos para ellos (Morris \& Morris 1966). En tanto que los animales son percibidos como individuos, las plantas son reconocidas en muchos casos sólo como parte del hábitat de los animales (Greaves et al. 1993; Hershey 1996). Por eso, cuando se le pide a los niños que mencionen plantas, aparecen respuestas indefinidas tales como "árboles", "flores", "plantas con espinas", "árboles del bosque" (por ejemplo Ashworth et al. 1995; Nates et al. 2010; Campos et al. 2012). En general, las plantas más apreciadas son aquellas con flores coloridas, grandes y perfumadas y con frutos carnosos y vistosos, como la rosa, la dalia, la margarita y las plantas frutales (Tunnicliffe 2001; Lindemann-Matthies 2005). Se ha propuesto, como explicación evolutiva, que estas características de las plantas fueron señales de disponibilidad de alimento para los hombres primitivos (Heerwagen \& Orians 1993).
A los niños les gustan los animales grandes, en particular aquellos que son similares a los humanos en apariencia y/o comportamiento, como los monos y los pandas, o a los que se les atribuyen cualidades antropomórficas, como los perros, delfines y caballos ("loveable animals" según Kellert 1985; Morris \& Morris 1966; Bell 1981; Morris 1982; Ward et al. 1998; Woods 2000; Lindemann-Matthies 2005). Algunas características como la presencia de ojos grandes, la capacidad de tomar una postura erguida, la manipulación de objetos, la afinidad y posibilidad de interacción con los humanos y la demostración de emociones y comportamientos inteligentes, dirigen las preferencias hacia determinadas especies (Kellert 1980, 1993; Schulz 1985). En general, son vertebrados grandes y carismáticos, en particular dentro del grupo de los mamíferos. Por el contrario, los reptiles, anfibios, peces e invertebrados son menos apreciados. A pesar de su importancia ecológica, algunos representantes de estos grupos despiertan aversión y miedo, se los relaciona con enfermedades o son considerados dañinos para los cultivos y el ganado, como las serpientes, arañas e insectos. En general, éstos son animales pequeños y diferentes a los humanos en morfología y comportamiento (Morris \& Morris 1966, 1965; Morris 1982; Kellert 1993, 1996).

En concordancia con lo expuesto, un estudio basado en 865 encuestas aplicadas a estudiantes de las escuelas rurales de Valle Fértil (San Juan) a quiénes se les preguntó

Tabla 1. Los diez animales que fueron mencionados con más frecuencia (expresado como \% de respuestas) cuando a los estudiantes rurales de la provincia de San Juan (n=865) se les preguntó qué animal les gustaba más, qué animal les gustaba menos, y cuál animal era el más útil para el hombre.

*: Animales nativos. Debajo: síntesis de resultados para taxa agrupados. Tabla adaptada de Nates et al. (2010).

Table 1. The ten animals that students $(n=865)$ liked most, disliked most, and perceived as most useful.

*: Native wild animal taxa. Below: summaries for all taxa named. Modified tabla from Nates et al. (2010).

\begin{tabular}{|c|c|c|c|c|c|}
\hline \multicolumn{2}{|l|}{ Animales que más gustan } & \multicolumn{2}{|l|}{ Animales que menos gustan } & \multicolumn{2}{|l|}{ Animales más útiles } \\
\hline Taxon & $(\%)$ & Taxon & $(\%)$ & Taxon & $(\%)$ \\
\hline Perro (Canis familiaris) & 23.4 & Gato (Felis silvestris) & 16.2 & Caballo (Equus ferus caballus) & 38.4 \\
\hline Caballo (Equus ferus caballus) & 23.2 & Serpientes (indeterminado)* & 9.2 & Perro (Canis familiaris) & 22.2 \\
\hline Gato (Felis silvestris) & 10.6 & Cerdo (Sus domesticus) & 6.2 & Burro (Equus asinus) & 10.8 \\
\hline conejo (Oryctolagus cuniculus) & 8.0 & Sapos (Bufo spp.)* & 6.2 & Vaca (Bos taurus) & 8.9 \\
\hline Puma (Puma concolor $)^{*}$ & 5.0 & Arañas (indeterminado)* & 5.7 & Gato (Felis silvestris) & 4.0 \\
\hline León (Panthera leo) & 3.4 & Zorro gris (Pseudalopex griseus)* & 4.7 & Cabra (Capra hircus) & 2.8 \\
\hline Tigre (Panthera tigris) & 2.5 & Rata (Rattus norvegicus) & 4.3 & Liebre (Lepus europaeus) & 1.7 \\
\hline Cabra (Capra hircus) & 1.8 & Perro (Canis familiaris) & 4.2 & Guanaco (Lama guanicoe)* & 1.3 \\
\hline Loros (Psitacidos spp.)* & 1.0 & Zorrino (Conepatus chinga)* & 4.0 & Zorro gris (Pseudalopex griseus)* & 0.9 \\
\hline Burro (Equus asinus) & 0.9 & Caballo (Equus ferus caballus) & 2.8 & Gallina (Gallus gallus) & 0.8 \\
\hline Exóticos domésticos & 72.2 & Exóticos domésticos & 36.5 & Exóticos domésticos & 89.2 \\
\hline Nativos & 15.6 & Nativos & 52.2 & Nativos & 10.0 \\
\hline Exóticos silvestres & 12.2 & Exóticos silvestres & 11.3 & Exóticos silvestres & 0.8 \\
\hline
\end{tabular}


cuál era su animal favorito, muestra que los preferidos son el perro y el caballo (Tabla 1; Nates et al. 2010). Estos animales también son considerados los más útiles: el perro como guardián de la casa y ayudante en las tareas de campo (para cuidar el ganado, cazar, etc.); el caballo como medio de transporte y carga. Los animales que resultan menos atractivos para los niños son el gato, las serpientes, el cerdo y los sapos (Tabla 1; Nates et al. 2010). Contrariamente a lo encontrado en otros estudios en los cuales el gato es uno de los animales más atractivos para los niños (Morris \& Morris 1966; Woods 2000), a los chicos rurales de San Juan probablemente no les guste este animal porque lo asocian con sus presas (ratas, ratones, serpientes, etc.) o consideran que es un animales difícil de controlar. Un poco más del $50 \%$ de las respuestas acerca de los animales menos apreciados por los niños menciona especies nativas, las cuales también son consideradas menos útiles que las exóticas domésticas (Tabla 1; Nates et al. 2010).

Para los estudiantes rurales de San Juan, la rosa es la planta preferida y la más útil (Tabla 2; Nates et al. 2010). Entre las plantas más apreciadas se mencionan especies ornamentales con flores vistosas y árboles frutales. Las especies nativas no son las que más gustan a los niños, sin embargo las consideran como las más útiles para preparar infusiones, para uso medicinal, para extraer leña y para producir sombra (Nates et al. 2010).

\section{CONOCIMIENTO ACERCA DE LA BIODIVERSIDAD}

El conocimiento de las personas acerca de la biodiversidad depende de diferentes factores socio-demográficos, como la procedencia (rural o urbana), la edad y el género. El conocimiento ecológico relacionado con el reconocimiento de las especies y sus usos se vincula de manera estrecha con el nivel de dependencia de los recursos naturales y la frecuencia de las interacciones con el ambiente. Por este motivo, algunos autores sostienen que las personas de países menos desarrollados y de comunidades rurales tienen más conocimientos acerca de la biodiversidad y del ambiente local (Chand \& Shulka 2003; Reyes-García et al. 2005; Pilgrim et al. 2007). Sin embargo, la creciente convergencia en los estilos de vida de poblaciones urbanas y rurales y el acceso a los medios masivos de comunicación pueden reducir las diferencias en el conocimiento ecológico (Lowe \& Pinhey 1982; Bogner \& Wiseman 1997; Berenger et al. 2005; Huddart-Kennedy et al. 2009).

La falta de conocimiento ecológico y ambiental que permita la participación activa y responsable en la toma de decisiones, sumada a un alejamiento de la naturaleza por parte de las personas, afectan de forma negativa el apoyo del público hacia la conservación de la biodiversidad (Miller 2005; Jordan et al. 2009; Bickford et al. 2012). Sin conocimiento ni contacto con los organismos que forman parte

Tabla 2. Las diez plantas que fueron mencionadas con más frecuencia (expresado como \% de respuestas) cuando a los estudiantes ( $n=865$ ) se les preguntó: a) qué planta que les gustaba más, b) qué planta les gustaba menos, y c) qué planta era la más útil para el hombre.

*: Plantas nativas. Debajo: síntesis de resultados para taxa agrupados. Tabla adaptada de Nates et al. (2010).

Table 2. The ten plants that students $(n=865)$ a) liked most, b) disliked most, and c) perceived as most useful.

*: Native wild plant taxa. Below: summaries for all taxa named. Modified tabla from Nates et al. (2010).

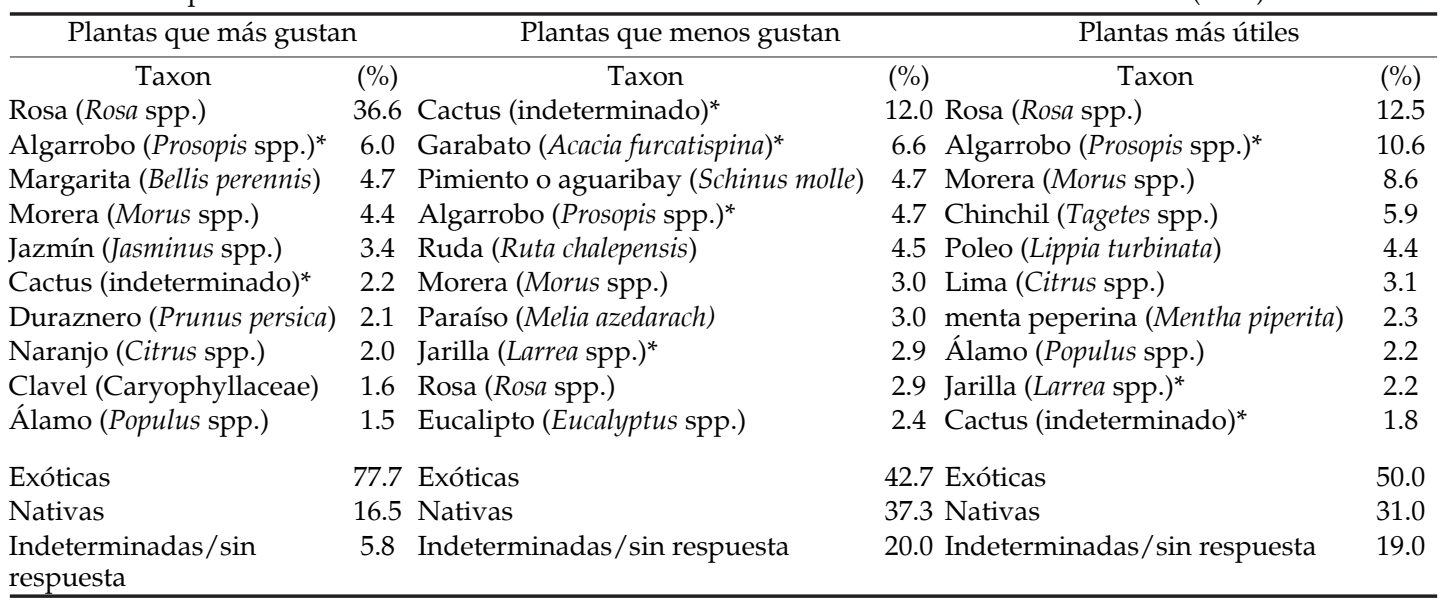


del ambiente inmediato, las personas no saben ni se preocupan por lo que les pueda ocurrir (Weilbacher 1993). Es necesario mejorar este conocimiento como un paso más para lograr cambios en las actitudes y comportamientos acerca de la biodiversidad y los problemas ambientales (Sudarmadi et al. 2001).

A pesar del interés y la curiosidad de los niños por los seres vivos, algunos estudios muestran que, en general, los niños tienen poco conocimiento sobre las plantas y los animales de su entorno inmediato (Ryman 1974; Wandersee 1986; Lindemann-Matthies 2002, 2006; Balmford et al. 2002; LindemannMatthies \& Bose 2008). Esta falta de conocimientos acerca de la biodiversidad ha sido atribuida, en parte, al poco tiempo que las escuelas invierten en actividades que involucren el contacto directo de las personas con la naturaleza (Pfligersdorffer 1991; Kinchin 1993; Barker et al. 2002; LindemannMatthies 2006). Los niños, especialmente los procedentes de zonas urbanas, están más familiarizados con especies que muchas veces no forman parte de la biodiversidad local y que conocen a través de fuentes como los libros, la televisión o internet (Trommer 1980; Hesse 1983; Lock 1995; Paraskevopoulos et al. 1998; Campos et al. 2012).

En un estudio realizado para determinar la familiaridad de los estudiantes de zonas urbanas y rurales de la provincia de Mendoza con la biodiversidad, se pidió a los alumnos ( $n=1746$; entre 7 y 18 años) mencionar 10 plantas y 10 animales e indicar dónde habían conocido a cada uno (e.g., en el campo, jardín de la casa, libro, televisión, internet, etc.). Fueron capaces de nombrar numerosas especies (casi 500). Como un resultado interesante, se encontró que, a diferencia de trabajos que muestran que los estudiantes conocen pocas especies de plantas y tienen dificultades para nombrarlas (Ryman 1974; Wandersee 1986; Lindemann-Matthies 2002), en las zonas rurales de San Juan y Mendoza los niños mencionan casi 200 especies vegetales (Nates et al. 2010; Campos et al. 2012). Sin embargo, ellos conocían más plantas y animales exóticos que nativos $\mathrm{y}$, como en el resto del mundo, estaban familiarizados con las mascotas, las plantas ornamentales y los mamíferos carismáticos. En particular, los animales y plantas con problemas de conservación tuvieron poca representación: sólo $0.5 \%$ de las respuestas incluyó a los guanacos y ñandúes, $0.6 \%$ al cóndor, $0.1 \%$ a la mara y $0.04 \%$ a las ratas cola de pincel (Campos et al. 2012).

Tal como se mencionó antes, la procedencia, el género y la edad de los alumnos afectan el conocimiento relacionado con la biodiversidad y el contacto inicial que tienen con las especies. Cuando se realizó el estudio con los alumnos rurales y urbanos de Mendoza, se esperaba encontrar que los estudiantes rurales estuvieran más familiarizados con las especies nativas y que el primer contacto con ellas haya sido a través de fuentes directas, como el campo o el jardín de la casa. Por otro lado, los alumnos urbanos conocerían más a las especies exóticas y utilizarían fuentes de conocimiento alejadas de la naturaleza, como los libros, internet y la televisión. Sin embargo, el efecto de la procedencia no fue directo y el género de los alumnos tuvo una importante fuerza explicativa: los varones rurales conocieron a las especies nativas y mencionaron las salidas al campo como principal fuente de contacto con la biodiversidad. Ellos nombraron más aves, insectos, reptiles y anfibios que las niñas. Las niñas, por su parte, estuvieron más familiarizadas con las especies exóticas y destacaron como fuente de conocimiento el jardín de sus casas (Campos et al. 2012). Las diferencias entre niños y niñas, sobre todo en ambientes rurales, puede explicarse por la división de roles: los varones ayudan a su padre con el trabajo de campo y las niñas colaboran con su madre en los quehaceres domésticos. Por esto los varones interactúan con especies nativas y las niñas conocen las especies cercanas al hogar, por ejemplo las plantas cultivadas en la huerta o el jardín de la casa (Nates et al. 2010). Las niñas, debido a una apreciación estética, generalmente están familiarizadas con las mascotas, los mamíferos carismáticos y las plantas con flores (Badarraco 1973). Esta actitud humanista también se refleja en mujeres adultas, quienes suelen manifestar miedo o poco interés por las especies silvestres (Kellert \& Berry 1987).

No obstante, ante el argumento de que las poblaciones rurales de las zonas áridas de Argentina conservan su conocimiento ecológico tradicional relacionado con las especies nativas y el uso desde tiempos anteriores a la conquista española (Abraham et al. 2009; Ladio \& Lozada 2009; Vilela et al. 2009), el estudio comparativo entre alumnos rurales y urbanos no encontró un efecto importante del 
lugar de procedencia sobre la familiaridad con las especies (Campos et al. 2012). Es probable que el conocimiento ecológico tradicional se preserve en poblaciones rurales aisladas que ocupan las zonas no irrigadas de Mendoza y San Juan, más que en poblaciones rurales relacionadas con los oasis donde prosperan cultivos de origen Mediterráneo como la vid y el olivo (Vilela et al. 2009). Las escuelas con las cuales se trabajó en el estudio de familiaridad con especies pertenecen a este último grupo.

Los niños pequeños aprenden sobre plantas $\mathrm{y}$ animales a través de fuentes de contacto no directo, como los libros, la televisión o internet (Campos et al. 2012). Esto puede tener al menos dos interpretaciones: quizás estén perdiendo la cercanía con el ambiente natural debido a cambios profundos en el estilo de vida [i.e., niños y adultos sedentarios que pasan más horas frente al televisor y la computadora, y a las pocas actividades en la naturaleza que desarrollan las escuelas y las familias (Louv 2005)]. Obien, puede ocurrir que el incremento en los últimos tiempos de la producción de programas educativos y documentales sobre biodiversidad y problemas ambientales haya aumentado la disponibilidad de una fuente de conocimiento que puede resultar muy atractiva (Sudarmadi et al. 2001). Si este último fuera el caso, cabría destacar la responsabilidad de los medios masivos de comunicación en la construcción de conocimientos, valoraciones y apreciaciones de los niños hacia la naturaleza.

Desde hace algunos años, estudios europeos manifiestan su preocupación por la disminución en la currícula escolar de contenidos relacionados con la biodiversidad. Atribuyen esta situación, al reciente dominio de temas fisiológicos, moleculares y genéticos en los programas de enseñanza de la biología (Yore \& Boyer 1997; Lindemann-Matthies 2002). Sin embargo, advierten que ante la actual aceleración en la pérdida de hábitats y su biodiversidad asociada resulta acuciante volver a lo que llaman "educación tradicional en biología". Surge entonces la necesidad de facilitar el acercamiento de los estudiantes a la biodiversidad nativa y su problemática de conservación, por lo que se considera importante la identificación de las especies, el estudio de su biología y comportamiento, la comprensión de sus papeles en el ecosistema y el entendimiento del efecto de las actividades humanas.

\section{INTERVENCIONES EDUCATIVAS}

Numerosos proyectos de investigación llevados a cabo en Argentina están acompañados por intervenciones educativas que buscan difundir el conocimiento acerca de las especies y los ecosistemas (e.g., Borghi 2004; Guichón 2006; Giannoni 2007) o que intentan rescatar los saberes de las poblaciones que viven más cercanas a la naturaleza e integrarlos con saberes científicos (Ladio \& Lozada 2003; Ladio et al. 2007; Lucherini \& Merino 2008).

En América Latina, los proyectos que contemplan la educación para la conservación de la biodiversidad son necesarios porque la Ecología y la Educación Ambiental aún no están suficientemente implementadas en la curricula oficial (González-Gaudiano 2007; Borgerhoff Mulder et al. 2009). En los últimos tiempos se están llevando a cabo diversos programas de educación en conservación con el objetivo de sensibilizar a los estudiantes en la protección y el manejo sustentable de las especies nativas (e.g., Rozzi et al. 2000; Borgerhoff Mulder et al. 2009; Ruiz-Mallen et al. 2009; Nates et al. 2012). En la mayoría de los casos, la efectividad de estas experiencias educativas no se evalúa (Walsh-Daneshmandi \& MacLachlan 2006; González-Gaudiano 2007), aunque investigaciones previas han mostrado una diversidad de métodos cuantitativos y cualitativos y aproximaciones participativas que proveen una información valiosa acerca de los resultados de las intervenciones sobre el conocimiento, las actitudes y los comportamientos ambientales (ver referencias en Ruiz-Mallen et al. 2009). En general, en América Latina las experiencias educativas relacionadas con la conservación de la biodiversidad son organizadas desde el ámbito no formal por fundaciones o instituciones que ofrecen visitas, actividades, salidas, etc. de corta duración. La efectividad de estas experiencias cortas es limitada y, mientras algunos encuentran que pueden promover cambios a largo plazo en los conocimientos (Falk \& Balling 1982; Bitgood 1989; Lindemann-Matthies \& Kamer 2006), otros proponen que realmente no mejoran las actitudes hacia el ambiente (Borgerhoff Mulder et al. 2009).

En el marco del proyecto "Conociendo para conservar la biodiversidad del Parque Provincial Ischigualasto (Sitio Patrimonio de la Humanidad) y zona de influencia: 
propuesta educativa para los docentes y la comunidad del Valle" (Giannoni 2007) se llevó a cabo la evaluación de la experiencia educativa consistente en talleres de un día de duración en las nueve escuelas de nivel primario de Valle Fértil (Nates et al. 2012). Los talleres propiciaron charlas participativas con material audiovisual acerca de especies nativas y exóticas y las problemáticas asociadas a la introducción de especies, reconocimiento de especies nativas y sus adaptaciones para la vida en ambientes áridos utilizando material de colecciones didácticas de fauna. También permitieron la realización de juegos ambientales en el patio de las escuelas. Considerando la continua demanda de los docentes por material didáctico que incluyeran conocimientos acerca de la biodiversidad local, se prepararon láminas, cuadernillos con contenidos y sugerencias didácticas y fichas de flora y fauna con el objetivo de que el proceso de enseñanza-aprendizaje de los contenidos abordados en el taller continúe en el aula junto con los maestros. Los talleres se desarrollaron en las escuelas, donde se mantuvieron las condiciones de aprendizaje habituales en escenarios conocidos para que los alumnos no perdieran su concentración por la novedad de nuevos ambientes (Anderson \& Lucas 1997; Falk \& Dierking 2000).

Con el propósito de evaluar el potencial efecto del taller sobre los conocimientos y actitudes de los alumnos hacia la biodiversidad se diseñó un cuestionario que los estudiantes debieron responder antes del taller, inmediatamente después y pasado un año de realizar dicho taller. Se utilizaron fotos de especies nativas y exóticas presentes en el sitio de estudio e imágenes de personajes de dibujos animados (Nates et al. 2012). Las especies nativas incluyeron animales con problemas de conservación asociados a la caza porque localmente se les considera competidores o depredadores del ganado (puma, guanaco y cóndor) y plantas vulnerables a los cambios actuales del ecosistema (algarrobo Prosopis flexuosa, chica Ramorinoa girolae y retamo Bulnesia retama). Las especies exóticas incluyeron animales introducidos que potencialmente compiten con especies nativas y contribuyen al sobrepastoreo y reducción de la biodiversidad (liebre europea Lepus europaeus, burros asilvestrados Equus asinus, ganado vacuno Bos taurus) y plantas introducidas para forestación que modifican el ecosistema (eucaliptus Eucalyptus spp., pimiento Schinus molle y tamarindo Tamarix gallica). Las imágenes de dibujos animados que se mostraron a los estudiantes fueron personajes de "Los Simpson" y se incluyeron para evaluar si los alumnos identificaban mejor a personajes de la fantasía que a especies de su entorno (Balmford et al. 2002; Nates et al. 2012). La evaluación de la experiencia educativa intentó detectar cambios en los conocimientos, a través de la identificación de especies y la explicación de adaptaciones de los organismos a los ambientes áridos, y en las percepciones, a través de preguntas relacionadas con la apreciación estética y la utilidad de las especies (Nates et al. 2012).

Los cuestionarios aplicados antes del taller mostraron que los animales exóticos domesticados y los personajes de dibujos animados fueron mejor identificados por los estudiantes que las especies nativas locales. La mitad de los alumnos presentó dificultades para mencionar al menos una adaptación de los organismos a la aridez, aunque pudieron nombrar con fluidez los usos locales de las especies, mostrando así la falta de conocimientos escolares en ecología y la amplia experiencia práctica en el uso de los recursos. En cuanto a la percepción de las especies, la liebre europea y el eucalipto se consideraron las más atractivas, aunque también mencionaron el guanaco y el algarrobo. El burro y la vaca se citaron como las especies más útiles para el hombre (Nates et al. 2012).

A corto plazo, la experiencia educativa tuvo un efecto positivo sobre la identificación de las especies, sobre todo de las plantas. Un año más tarde, las especies que fueron familiares para los alumnos desde antes del taller, continuaron siendo bien identificadas. Sin embargo, el reconocimiento de las especies no familiares para los estudiantes y sobre las cuales habían aprendido en el taller se perdió a largo plazo. Lo mismo ocurrió con los contenidos que se habían aprendido en el taller acerca de las adaptaciones. Los niños mencionaron más adaptaciones inmediatamente después del taller. Por último, sólo en el corto plazo, especies no queridas (como el cóndor) tuvieron apreciaciones más positivas que antes del taller.

Este estudio mostró que una experiencia educativa corta puede ser significativa para los estudiantes si la información provista es sencilla y se relaciona de alguna manera 
con experiencias previas. Seguramente los resultados serán más notables cuando las intervenciones involucren visitas frecuentes a las escuelas y actividades participativas que incluyan aspectos emocionales (Knapp \& Poff 2001; Borgerhoff Mulder et al. 2009; Ruiz-Mallen et al. 2009).

\section{CONCLUSIONES}

Los resultados mostraron que la percepción y la apreciación hacia la biodiversidad por los estudiantes de zonas áridas de Argentina están dirigidas en general a los animales domésticos y las plantas ornamentales. Los estudiantes aprenden acerca de la biodiversidad a través de fuentes como el jardín de la casa, el campo y las visitas al zoo.

Si bien se esperaba encontrar que los alumnos rurales estuvieran más familiarizados con las especies nativas, los estudios mostraron una valoración utilitaria de la naturaleza y, aunque los varones rurales son los alumnos que más conocen la biodiversidad nativa, las preferencias están dirigidas a las especies domésticas utilizadas en su vida cotidiana.

Si bien es importante que los estudiantes aprecien y conozcan la biodiversidad en general, resulta necesario que adquieran más familiaridad con las especies nativas y con los ambientes naturales para que valoren su conservación. Para ello, la educación es una estrategia clave $y$, si se pretende lograr cambios a largo plazo en los conocimientos, actitudes, apreciaciones y valoraciones acerca de la biodiversidad, es necesario aumentar el esfuerzo junto a las escuelas, las familias, las organizaciones no gubernamentales, mediante intervenciones educativas con mensajes simples y claros y con actividades que se mantengan en el tiempo e impliquen el contacto directo con la naturaleza.

Agradecimientos: Agradecemos al grupo INTERBIODES (Interacciones Biológicas del Desierto), al GIB (Grupo de Investigaciones de la Biodiversidad) y a la Universidad de Zürich todo el apoyo brindado. CM Campos es investigadora de CONICET y este trabajo es fruto de las interacciones colaborativas con el Grupo de Percepción de la Biodiversidad y Educación Ambiental (Universidad de Zürich, Suiza).

\section{BiBLIOGRAFÍA}

Abraham, E; HF del Valle; F Roig; L Torres; JO Ares; et al. 2009. Overview of the geography of the Monte Desert biome (Argentina). J. Arid Environm., 73:144-153.
Anderson, D \& KB Lucas. 1997. The effectiveness of orienting students to the physical features of a science museum prior to visitation. Res. Sci. Ed., 27:485-495.

Ashworth, S; E Boyes; R Paton \& M Stanisstreet. 1995. Conservation of endangered species: what do children think? J. Environ. Educ. Inform., 14:229-244.

BADARRACO, RJ. 1973. Squirrels, or sunflowers? Am. Biol. Teach., 35:528-538.

Baird, JH; R Lazarowitz \& V Allman. 1984. Science choices and preferences of middle and secondary school students in Utah. J. Res. Sci. Teach., 21:47-54.

BAlmford, A; L ClegG; T Coulson \& J TAylor. 2002. Why conservationists should heed Pokemon. Science 295:2367.

BARKER, S; DSLINGSBY \& S TILLING. 2002. Ecological fieldwork: Is there a problem? Environm. Educ., 71:9-10.

Barquez, RM; M Díaz \& RA Ojeda. 2006. Mamíferos de Argentina: sistemática y distribución. Sociedad Argentina para el Estudio de los Mamíferos. Argentina. Pp. 359.

BELL, BF. 1981. When is an animal, not an animal? J. Biol. Educ., 15:213-218.

BerEnger, J; JA CoRraliza \& R MARTín. 2005. Rural-urban differences in environmental concern, attitudes, and actions. Eur. J. Psychol. Assess., 21:128-138.

BICKFORD, D; MRC PoSA; L QIE; A CAMPOS-ARCeiz \& EP Kudavidanage. 2012. Science communication for biodiversity conservation. Biol. Conserv., 151:74-76.

BiтGOod, S. 1989. School field trips: an overview. Visitor Behav., 4:3-6.

BOGNER, FX \& MWISEMAN. 1997. Environmental perception of rural and urban pupils. J. Environm. Psychol., 17:111-122.

BorgerhofF MuldER, M; R SCHACht; T CAROB; J SCHACHT \& B CARO. 2009. Knowledge and attitudes of children of the Rupununi: implications for conservation in Guyana. Biol. Conserv., 142:879-887.

BORGHI, CE. 2004. Biodiversidad de Fauna Silvestre de la zona de in uencia del Parque Nacional de San Guillermo: situación de conservación, usos actuales y propuestas de manejo sustentable. Experimentos piloto de uso no consuntivo con fines educativos y turísticos. Administración de Parques Nacionales. Argentina.

Campos, CM; S Greco; JJ Ciarlante; M Balangione; JB BENDER; ET AL. 2012. Students' familiarity and initial contact with species in the Monte desert (Mendoza, Argentina). J. Arid Environm., 82:98-105.

COLTON, TF \& P ALPERT. 1998. Lack of public awareness of biological invasions by plants. Nat. Area J., 18:262-266.

Czech, B; PR Krausman \& R Borkhataria. 1998. Social construction, political power, and the allocation of benefits to endangered species. Conserv. Biol., 12:1103-1112.

CHAND, VS \& SR SHULKa. 2003. 'Biodiversity contests': indigenously informed and transformed environmental education. Appl. Environm. Educ. Commun., 2:229-236.

CITES. Convention on International Trade in Endangered Species of Wild Fauna and Flora. 2011. www.cites.org/ eng/app/appendices.shtml (último acceso: 30711/2012).

DíAz, GB \& RA OJEDA (eds.). 2000. Libro Rojo de Mamíferos Amenazados de la Argentina. Sociedad Argentina para el Estudio de los Mamíferos. Argentina.

FALK, JH \& JD BALLING. 1982. The field trip milieu: learning and behavior as a function of contextual events. J. Educ. Res., 76:22-28.

FALK, JH \& LD DierKING. 2000. Learning from museums: visitor experiences and the making of meaning. AltaMira Press. USA. 
FlanNery, MC. 1991. Considering plants. Am. Biol. Teach., 53:306-309.

García Fernández, JJ; RA OJedA; RM Fraga; GB Díaz \& RJ Baigún. 1997. Libro Rojo de Mamíferos y Aves Amenazados de la Argentina. Fundación para la Conservación de las Especies y el Medio Ambiente, Sociedad Argentina para el Estudio de los Mamíferos y Asociación Ornitológica del Plata. Argentina.

GIANNONI, SM. 2007. Conociendo para conservar la biodiversidad del Parque Provincial Ischigualasto (Sitio Patrimonio de la Humanidad) y zona de in uencia: propuesta educativa para los docentes y la comunidad del Valle. Secretaría de Extensión, Vinculación y Servicios al Medio de la Universidad Nacional de San Juan. Argentina.

Giraudo, AR; V Arzamendia; GP Bellini; CA Bessa; CC Calamante; et Al. 2012. Categorización del estado de conservación de las Serpientes de la República Argentina. Cuad. Herpetol., 26(Supl. 1):303-326.

Gonzalez-Gaudiano, E. 2007. Educación ambiental: trayectorias, rasgos y escenarios. Plaza y Valdés y Universidad Autónoma de Nuevo León. Mexico.

Greaves, E; M Stanisstreet; E Boyes \& T Williams. 1993. Children's ideas about rainforests. J. Biol. Educ., 27:189-194.

GUICHÓN, ML. 2006. Educación, difusión y gestión como herramientas para alertar sobre la introducción de especies, tenencia responsable de mascotas y tráfico de fauna: ardillas exóticas como ejemplo regional. Secretaría de Extensión, Vinculación y Servicios al Medio de la Universidad Nacional de Luján. Argentina.

HeERWAGen, JH \& GH ORIANs. 1993. Humans, habitats, and aesthetics. Pp. 138-172 en: Kellert, SR \& EO Wilson (eds.). The Biophilia hypothesis. Island Press. Washington. USA.

HERSHEY, DR. 1996. A historical perspective on problems in botany teaching. Am. Biol. Teach., 58:340-347.

Hesse, M. 1983. Artenkenntnis bei Studienanfängern [Taxonomic knowledge of first-year students]. Der Biologieunterricht, 19:94-100.

Huddart-Kennedy, E; TM Beckley; BL McFarlane \& S NADEAU. 2009. Rural-urban differences in environmental concern in Canada. Rural Sociol., 74:309-329.

KELLERT,SR. 1980. American's attitudes and knowledge of animals. Wildlife Management Institute.Washington. USA.

KeLLERT, SR. 1985. Attitudes toward animals: age-related development among children. J. Environ. Educ., 16:29-39.

Kellert, SR \& JK Berry. 1987. Attitudes, knowledge, and behaviours toward wildlife as affected by gender. Wildlife Soc. B, 15:363-371.

KELLERT, SR. 1993. The biological base for human values of nature. Pp. 43-69 en: Kellert, SR \& EO Wilson (eds.). The Biophilia hypothesis. Island Press. Washington. USA.

Kellert, SR. 1996. The value of life. Island Press. Washington. USA.

KINCHIN, IM. 1993. Teaching ecology in England and Wales- A survey of current practice. J. Biol. Educ., 27: 755-760.

KINCHIN, IM. 1999. Investigating secondary-school girls' preferences for animals or plants: a simple 'head-tohead' comparison using two unfamiliar organisms. J. Biol. Educ., 33:95-99.

KNAPP, D \& R Poff. 2001. A qualitative analysis of the immediate and short-term impact of an environmental interpretive program. Environ. Educ. Res., 7:55-65.

Jordan, R; F Singer; J VAughan \& A BerKowitz. 2009. What should every citizen know about ecology? Front. Ecol. Environ., 7:495-500.

LAdIO, A \& M LozadA. 2003. Comparison of wild edible plant diversity and foraging strategies in two aboriginal communities of northwestern Patagonia. Biodivers. Conserv., 12:937-951.

Ladio, A; M Lozada \& M Weigandt. 2007. Comparison of traditional wild plant knowledge between aboriginal communities inhabiting arid and forest environments in Patagonia, Argentina. J. Arid Environm., 69:695-715.

LADIO, AH\& MLOZADA. 2009. Human ecology, ethnobotany and traditional practices in rural populations inhabiting the Monte region: resilience and ecological knowledge. J. Arid Environm., 73:222-227.

Lindemann-Matthies, P. 2002. The influence of an educational program on children's perception of biodiversity. J. Environ. Educ., 33:22-31.

LindeMANN-MatTHIES, P. 2005. 'Loveable' mammals and 'lifeless' plants: how children's interest in common local organisms can be enhanced through observation of nature. Int. J. Sci. Educ., 27:655-677.

LindeMANN-MatthiES, P. 2006. Investigating Nature on the Way to School: responses to an educational programme by teachers and their pupils. Int. J. Sci. Educ., 28:895-918.

Lindemann-MatthiEs, P \& E Bose. 2008. How many species are there? Public understanding and awareness of biodiversity in Switzerland. Hum. Ecol., 38:731-742.

Lindemann-Matthies, P \& T Kamer. 2006. The influence of an interactive educational approach on visitors' learning in a Swiss zoo. Sci. Educ., 90:296-315.

Lock, R. 1995. Biology and the environment- A changing perspective? Or "there's wolves in them there woods!" J. Biol. Educ., 29:3-4.

Louv, R. 2005. Last child in the woods. Saving our children from nature-deficit-disorder. Algonquin Books of Chapel Hill. North Carolina. USA.

LOWE, GD \& TK PINHEY. 1982. Rural-urban differences in support for environmental protection. Rural Sociol., 47: 114-128.

LucherinI, M \& MJ Merino. 2008. Perceptions of humancarnivore conflicts in the High Andes of Argentina. Mt. Res. Dev., 28:81-85.

Miller, JR. 2005. Biodiversity conservation and the extinction of experience. Trends Ecol. Evol., 20:430-434.

Montgomery, CA. 2002. Ranking the benefits of biodiversity: an exploration of relative values. J. Environ. Manage., 65:313-326.

Morris, D. 1982. Der Mensch mit dem wir leben [Manwatching]. Droemersche Verlagsanstalt. Munich, Alemania.

Morris, R \& D Morris. 1965. Men and snakes. Hutchinson \& Co. London. UK.

Morris, R \& D Morris. 1966. Men and pandas. Sphere Books Limited. London. UK.

Nates, J; C Campos \& P Lindemann-Matthies. 2010. Students' perception of plant and animal species: a case of study from rural Argentina. Appl. Environm. Educ. Commun., 9:131-141.

NATES, J; C CAMpos \& P LindEMANN-MATtHIES. 2012. The impact of a shortconservation education workshop on Argentineans students' knowledge about and attitudes towards species. Eurasia J. Math. Sci. E Tech. Ed., 8:257-267.

OjedA, RA \& GB DíAz. 1997. La categorización de los 
mamíferos de Argentina. Pp. 73-163 en: García, FJ; RA Ojeda; RM Fraga; GB Díaz \& RJ Baigún (eds.). Libro Rojo de los mamíferos y aves amenazados de Argentina. Administración de Parques Nacionales, Buenos Aires.

Paraskevopoulos, S; S Padeliadu \& K Zafiropoulos. 1998. Environmental knowledge of elementary school students in Greece. J. Environ. Educ., 29:55-60.

Pfligersdorffer, G. 1991. Die biologisch-ökologische Bildungssituation von Schulabgängern [Biological and ecological knowledge of school-leavers]. Abakus Verlag. Salzburg, Austria.

PimBert, MP \& JN PRETTY. 1995. Parks, people and professionals putting participation into protected area management. UNRISD Discussion Paper 57. UNRISD-IIED-WWF. Ginebra, Suiza.

Pilgrim, SE; C Cullen C; DJ Smith \& J Pretty. 2007. Ecological knowledge is lost in wealthier communities and countries. Environ. Sci. Technol., 42:1004-1009.

PoOLEY, JA \& MO'CONNOR. 2000. Environmental education and attitudes: emotions and beliefs are what is needed. Environ. Behav., 32:711-723.

Prado, WS; T Waller; DA Albareda; MR Cabrera; E ETCHEPARE; ET AL. 2012. Categorización del estado de conservación de las tortugas de la República Argentina. Cuad. Herpetol., 26(Supl. 1):375-387.

Reyes-García, V; V Vadez; T Huanca; W Leonard; D WILKIE. 2005. Knowledge and consumption of wild plants: a comparative study in two Tsimane' villages in the Bolivian Amazon. ERA Ethnobotany Research and Applications, 3:201-207.

Rozzi, R; JR Silander; JJ Armesto; P Feinsinger \& F MASSARDO. 2000. Three levels of integrating ecology with the conservation of South American temperate forests: the initiative of the Institute of Ecological Research Chiloé, Chile. Biodivers. Conserv., 9:1199-1217.

Ruiz-Mallen, I; L Barraza; B Bodenhorn \& V ReyesGARCíA. 2009. Evaluating the impact of an environmental education programme: an empirical study in Mexico. Environ. Educ. Res., 15: 371-387.

RYMAN, D. 1974. Children's understanding of the classification of living organisms. J. Biol. Educ., 8:140-144.

SChacter, DL; DT Gilbert \& DM WeGner. 2011. Psychology. Worth Publishers. New York. USA.

SudARMAdI, S; S SuZUKI; T KaWAdA; H NetTI; S SOEMANTRI; ET AL. 2001. A survey of perception, knowledge, awareness, and attitude in regard to environmental problems in a sample of two different social groups in Jakarta, Indonesia. Environ. Dev. Sustain., 3:169-183.
TABENI, S \& RA OJEDA. 2005. Ecology of the Monte Desert small mammals in disturbed and undisturbed habitats. J. Arid Environm., 63:244-255.

Tisdell, C; C Wilson \& HS Nantha. 2006. Public choice of species for the 'Ark': Phylogenetic similarity and preferred wildlife species for survival. J. Nat. Conserv., 14:97-105.

TrombulaK, SC; KS Omland; JA Robinson; JJ LusK; TL FleISCHNER; ET AL. 2004. Principles of conservation biology: recommended guidelines for conservation literacy from the Education Committee of the Society for Conservation Biology. Conserv. Biol., 18:1180-1190.

Trommer, G. 1980. Naturschutz im BiologieunterrichtBegriffsbilder bei Schülern der Primarstufe und Sekundarstufe I [Conservation in biology educationIdeas of primary and secondary school students]. Verhandlungen der Gesellschaft für Ökologie, 8:503-508.

TunNICLIFFE, SD. 2001. Talking about plants-comments of primary school groups looking at plant exhibits in a botanical garden. J. Biol. Educ., 36:27-34.

Vertch, CR \& MN Clout. 2001. Human dimensions in the management of invasive species in New Zealand. Pp. 63-71 en: McNeely, JA (ed.). The Great Reshuf ing: Human Dimensions of Invasive Alien Species. IUCN.

Vilela, A; ML Bolkovic; P Carmanchahi; M Cony; D de LAMO; ET AL. 2009. Past, present and potential uses of native flora and wildlife of the Monte Desert. J. Arid Environm., 73:238-243.

Walsh-Daneshmandi, A \& M MacLachlan. 2006. Toward effective evaluation of environmental education: Validity of the children's environmental attitudes and knowledge scale using data from a sample of Irish adolescents. J. Environm. Educ., 37:13-23.

WANDERSEE, JH. 1986. Plants or animals-which do junior high school students prefer to study? J. Res. Sci. Teach., 23:415-426.

WANDERSEE, JH \& EE SChussLer. 1999. Toward a theory of plant blindness. Plant. Sci. Bul., 47:2-9.

WARD, PI; N MOSBERger; C KistLer \& O FisCher. 1998. The relationship between popularity and body size in zoo animals. Conserv. Biol., 12:1408-1411.

WeILBACHER, M. 1993. The renaissance of the naturalist. J. Environ. Educ., 25:4-7.

Woods, B. 2000. Beauty and the beast: preferences for animals in Australia. J. Tourism St., 11:25-34.

YORE, LB \& S BOYER. 1997. College students' attitudes towards living organisms: The influence of experience \& knowledge. Am. Biol. Teach., 59:558-563. 